\title{
Review: some psychosocial interventions can help children and families cope with chronic health conditions
}

\author{
Bauman LJ, Drotar D, Leventhal JM, et al. A review of psychosocial interventions for children with chronic health conditions. \\ Pediatrics 1997 Aug;100:244-51.
}

\section{Objective}

To evaluate the effectiveness of psychosocial interventions for children with chronic health conditions.

\section{Data sources}

English language studies published in peer reviewed journals were identified by searching Medline and Psychological Abstracts from 1979-93. Additional studies were identified by scanning the bibliographies of retrieved articles and by contacting experts.

\section{Study selection}

Studies were selected if the intervention targeted children with a chronic health condition or their family members; a planned psychosocial intervention was evaluated (naturally occurring family resources, medical interventions and medical or physical treatment, medications, or treatment regimens were excluded); psychological or social outcomes were examined; and $\geqslant 15$ participants were included in the study which had random assignment to treatment groups, a matched comparison group, or a convenience comparison group.

\section{Data extraction}

Data were extracted on type and intensity of the intervention, target group, extent of programme integration with the child's medical care, level of training of the intervener, consistency of the delivery of the intervention, the extent to which a theoretical model was used to develop the intervention, study design, sample size, patient baseline characteristics, follow up, and outcome measures.

\section{Main results}

266 studies were identified. 15 studies met the selection criteria and reported the results of separate programmes. Studies evaluated psychosocial interventions in children with asthma $(n=7)$, cancer $(n=3)$, epilepsy $(n=2)$, and mixed diagnoses $(n=3)$. A meta-analysis was not done because the studies were too heterogeneous. 10 studies were randomised controlled trials. Overall, 11 studies showed a positive effect on at least 1 psychosocial outcome, and 4 studies showed mixed results. Several types of specific psychosocial outcomes were reported in these studies: psychiatric or behavioural symptoms, self esteem, self worth and social competence, locus of control, and family functioning. Psychiatric or behavioural symptoms were substantially affected most often. 8 studies used standardised psychiatric or behavioural symptom scales and in 6 studies the intervention led to a statistically significant positive effect. 9 studies measured effects on self esteem, self efficacy, or social competence and of these, 4 reported statistically significant improvements. Locus of control was examined in 3 studies; 1 showed a positive effect, and 2 showed no effect. 7 studies included measures of other psychological or social dimensions including stress, protectiveness, social support, and satisfaction, and several examined illness related anxiety or fear. Only 3 studies found positive effects in any of these domains. Of 3 studies that examined family functioning, 1 reported a substantial improvement with psychosocial intervention.

\section{Conclusions}

Some psychosocial interventions can help children and families cope with the psychological and social consequences of chronic health conditions. However, there is a need for more methodologically rigorous evaluations.

Sources of funding: William T Grant Foundation and Maternal and Child Health Bureau.

For article reprint: $\operatorname{Dr}$ L J Bauman, Albert Einstein College of Medicine, 1300 Morris Park Avenue, NR 7 South 21,Bronx, NY 10461, USA. Fax +17189184388.

Abstract modified from one published in Evidence-Based Nursing 1998 Apr.

\section{Commentary}

Bauman et al provide good evidence that psychosocial interventions for children with chronic conditions can make a difference. Two issues are worthy of brief consideration by clinicians and researchers. Firstly, eligible studies were selected partly on the basis that they reported a planned evaluation of a psychosocial intervention. It is possible that articles excluded from this review (eg, those describing other medical interventions or evaluations of perceptions of service delivery) might have contained important evidence on this topic. For example, a report by King et al describes correlations between parents' perceptions of services that they receive for their child with a neurodevelopmental disability and their increased satisfaction with services and decreased stress in dealing with their child's treatment programme. ${ }^{1}$ Thus, it is likely that evidence reported here is a conservative estimate of the real effect that services and programmes can have on the populations of interest.

Secondly, what is unclear from this review is the effect, if any, that the studies included in this review have had on clinical thinking or practice. The authors have not addressed this dimension of the dissemination and uptake challenge. Increasing interest in the issues of dissemination and uptake of new information (National Center for the Dissemination of Disability Research ${ }^{2}$ ), may prompt future reviewers (and indeed, primary researchers) to assess the real effect of research findings (using the forward tracking capacity of the Science Citation Index, among others) and to address the reasons why sound and relevant discoveries take so long to become part of standard practice. It would be interesting to determine, for example, how many of the recent studies included in this review referred to and built upon earlier work.

Programme evaluation is a formidable task, the steps of which are well outlined by Bauman et al. One can only hope that their expectations of clinician researchers will be endorsed by funders to support the complex work required to execute programme evaluation in a systematic and scientifically credible way.

Peter Rosenbaum, MD, FRCP(C) McMaster University Hamilton, Ontario, Canada

1 King SM, Rosenbaum PL, King G. Parents' perceptions of caregiving: development and validation of a measure of process. Dev Med Child Neurol 1996;38:757-72.

2 National Center for the Dissemination of Disability Research. A review of the literature on dissemination and knowledge utilization. Bethesda: National Institute on Disability and Rehabilitation Research, 1996. 